\title{
Internal and External Encoding Style and Social Motivation
}

\author{
Pawel Lewicki \\ University of Tulsa
}

The wide variety of social motivations that we encounter in everyday life is due partially to different conditions in which the observed people find themselves (i.e., to situational factors). However, it is probably almost trivial to state that the main reason for that observed "variety" is that the people we observe simply "differ from each other."

If so, then it is surprising to realize that research on social motivation usually deals with the nature of the proposed explanatory processes which are assumed to work similar across individuals, and it appears that very little research attention is devoted in this context to individual differences, even though they account probably for the majority of the variance of what research on social motivation (in general) is trying to explain.

One of possible reasons for this paradox is the apparent lack of success of the traditional research on individual differences to offer sufficiently "deep" explanations (i.e., explanations in terms of the underlying processes) and its apparent preoccupation with the relatively simple taxonomies that have been typically mostly descriptive (as opposed to explanatory) in nature. The traditional research on individual differences has been producing more benefits for the applied areas (e.g., personnel selection in I/O psychology) than for understanding the general determinants of social motivation.

One of the potential sources of new relevant evidence that could help understand the nature (i.e., underlying processes) of social motivation is research on implicit cognition and the processes of acquisition of knowledge that cannot be articulated by the perceivers. As summarized by Greenwald \& Banaji (1995):

"Much social cognition occurs in an implicit mode. (...) The missing ingredient is now available, as cognitive psychologists have succeeded in producing several varieties of unconscious cognition reliably in the laboratory (see overviews by Greenwald, 1992; Kihlstrom, 1987, Lewicki, 1992), and investigations of implicit social cognition are well underway. (...) Perhaps the most significant remaining challenge is to adapt these methods for efficient assessment of individual differences in implicit social cognition." (p. 20).

There has been substantial progress made in understanding the implicit cognition and now we know more about some of the processes of nonconscious acquisition of knowledge. Also, there are reasons to believe that these processes are a major driving force creating differences between individuals and especially differences in their schemata - which act as crucial filters limiting what a person can "notice" (encode) and determining the subsequent dynamics of the further development of the implicitly acquired knowledge. 
The present paper summarizes a line of research that resulted from this very research tradition (studies on nonconscious acquisition of knowledge) and which has produced consistent findings pointing to one of the mechanisms of individual differences that can explain differences in social motivation.

The starting point of this line of research was an entirely unexpected observation made when we were looking closely at the results of some experiments on self-perpetuation, conducted almost 10 years ago We need to start with some background information on that previous research.

\section{Cognitive Self-perpetuation in Encoding}

The phenomenon of self-perpetuation explains how individuals may sometimes acquire cognitive (encoding/interpretive) dispositions that are based on relations between variables which are not (statistically) inter-related in the real world, and relations which do not reflect what these individuals have been encountering. It can also explain how such dispositions can gradually strengthen and "grow" over time in the apparent absence of any supportive evidence.

This cognitive self-perpetuation may on the surface resemble the phenomenon of the social "self-fulfilling prophecy." Unlike self-fulfilling prophecy, however, it does not require any active interactions with the outside world or "causing" the outside world to respond in the way that the individual is expecting. Instead - in the cognitive self-perpetuation - the confirmations of the expectations are caused entirely by a bias in encoding. The mechanism of cognitive self-perpetuation works as follows.

The specific mechanism. If an individual exhibits even a very weak (and not articulated) encoding bias implying a relation between two variables A and B (e.g., "people with dark eyes (A) - are arrogant (B)"), then any subsequent encounters with A ("dark eyes") in the absence of unambiguous information about B ("arrogance") may bias the encoding of the (objectively ambiguous) level of $\mathrm{B}$ in the direction that is consistent with the initial A-B relation. This may artificially generate experience that is functionally equivalent of encountering "real" instances supportive of the A-B relation, and as a result of this simple process of "filling in the blanks" (or converting ambiguities into what is expected), the strength of the schema assuming the A-B relation can gradually grow over time despite the absence of any objectively supportive evidence.

Generality. This phenomenon appears to be quite ubiquitous. It has been demonstrated with a wide variety of stimulus materials and experimental conditions in over 50 experiments, and it may be responsible for the development of a wide variety of those individual differences which cannot be explained by the more straightforward forms of explicit and implicit "learning" about the environment, or by the genetic factors (Gill, 2000; Hill, Lewicki, \& Czyzewska, \& Boss, 1989, Hill, Lewicki, \& Czyzewska, \& Schuller, 1990; Hill, Lewicki, \& Neubauer, 1991; Lewicki, Hill, \& Czyzewska 1992, 1994; Lewicki, Hill, Sasaki, 1989; Stamov-Rossnagel, 2001; Vequist, 2001). Self-perpetuation can contribute to the development of a wide variety of personality dispositions from simple ones, such as preferences for colors, music, art, landscapes, interest in specific subjects, activities, attraction to specific types of people, etc. to the development of disorders. 
Specifically, there are reasons to believe that cognitive self-perpetuation-like mechanisms may play a significant role in the gradual deepening of interpretive biases so often observed in a variety of disorders, from Paranoia (Combs, in press) to Anxiety Disorders and Depression (Hill, Lewicki, \& Neubauer, 1991).

At the same time, both theoretical reasoning and empirical data suggest that selfperpetuation may act as a "catalyst," accelerating the process of development of new interpretive schemata, and facilitate acquisition of skills (Gill, 2000; Lewicki, Hill, \& Czyzewska 1992, Sailer \& Grubb, 1999; Vequist, 2001).

Individual differences. A careful analysis of the individual results of participants in these numerous laboratory experiments on self-perpetuation revealed clear individual differences. Namely, while self-perpetuation appears to be a general phenomenon that can be observed to some extent in most or all individuals, its dynamics (or "propensity to selfperpetuate") turned out to vary significantly across individuals: In some of them, the encoding indicative of self-perpetuation represents only a slight bias in their perception, while in others, it is strong, and it progresses very rapidly. Moreover, these differences were not related to the ease of the initial acquisition of the covariation (A-B) to be selfperpetuated in the experiment, or to other potential indices of attention or the motivation to participate in the study.

Also, these individual differences appeared to be stable across experiments when the same individuals participated in more than one experimental procedure.

One aspect of these differences was identified in studies in which at one point of the procedure, the covariation present in the stimulus material was changed. It turned out that participants differ in their ability to abandon an encoding disposition when it appears to be inadequate, and to switch to a new one. For example, the analysis of changes in participants' performance across segments of a task that requires "switching" of encoding algorithms (and the qualitative analysis of the "false alarm" errors) has revealed that while some participants easily "accept" the inappropriateness of the current encoding algorithm, stop applying it, and start developing a new one, other participants appear less sensitive to the changing conditions (and more reliant on the existing disposition) and continue to almost "compulsively" - act on the previously developed encoding algorithm despite its lack of fit to the new segment of the material.

\section{Styles of Cognitive Encoding}

Because these systematic differences in behavior suggested by these analyses may pertain to some, potentially very general, low-level aspects, or styles of encoding, we realized that understanding the cause (or the mechanism) of these differences could bring significant insights about the mechanisms of human cognition.

\section{Explanation of individual differences in self-perpetuation: The "threshold of instantiation of schemata" hypothesis. We hypothesized that the observed individual differences in the tendency to self-perpetuate may result from individual differences in more elementary processes of encoding.}

Specifically, as it is widely accepted in cognitive psychology, encoding processes impose on stimuli preexisting categories (i.e., interpretive schemata) even if the stimuli do not 
match very well the categories. This process of imposing such imperfectly fitting interpretive schemata has been shown in a number of studies on pattern recognition and prototype abstraction (e.g., Posner, et al., 1967, Reed, 1972), person perception (e.g. Cantor \& Mischel, 1979; Higgins \& King, 1981), and, more recently, in research on the nonconscious acquisition of information about covariations and its influence on subsequent encoding processes (Lewicki, 1986a, 1986b; Lewicki, Czyzewska, \& Hoffman, 1987; Lewicki, Hill, \& Bizot, 1988).

Even though, in order for an interpretive schema to be imposed on the stimulus, the match between the stimulus and the schema does not have to be perfect, there always needs to be a particular minimum amount of direct or indirect evidence (supportive of selecting the particular interpretive schema) available to the perceiver before the schema can be instantiated. Theoretically, the "required" threshold amount of such evidence (necessary to instantiate an interpretive schema) can represent a trade-off between speed and accuracy: The lower the threshold (i.e., the minimum amount of evidence) necessary to trigger the use of an encoding schema, the faster the encoding (at the expense of accuracy - because the hastily selected schema may not be the most appropriate one). On the other hand, the higher the threshold (i.e., the minimum amount of evidence) necessary to trigger the instantiation of an encoding schema, the more accurate the selection of the schema (at the expense of speed).

"Internal" vs. "External" encoding style. In summary, the hypothesis that lead to the development of this line of research, states that the observed individual differences in the tendency to self-perpetuate (which were found to be relatively consistent across procedures) are at least partially due to the differences between participants in terms of how "hasty" (or "internal" -- i.e., based on internal encoding schemata) vs. conservative (or "external" -- based on data from external stimuli) their encoding processes are.

Put another way, the hypothesized dimension of the encoding style (theoretically spanning from very "Internal" - fast but relying excessively on expectations, to very "External" compulsively attached to the external evidence, and thus slow) determines how much supportive evidence a perceiver needs to collect before imposing an interpretive schema on a stimulus (i.e., perceiving the stimulus as an instantiation of the schema).

The more "internal" the style of encoding, the greater the probability that the environmental cues would be interpreted in terms of preexisting ("internal") encoding schemata, thus providing support for those schemata and contributing to their reinforcement through the process of self-perpetuation, as discussed in previous sections.

\section{Empirical Evidence for Styles of Cognitive Encoding}

In a series of preliminary studies, we constructed a questionnaire to identify the hypothesized behavioral "symptoms" of the Internal (i.e., non-conservative, "hasty") encoding style, and then correlated participants' responses to that questionnaire first with:

(a) the patterns of response times and responses in the cognitive (computer controlled) tasks used to identify and measure self-perpetuation, and then

(b) other tasks designed to test the expected behavior of Internal and External encoders. 
Experiencing the split-second illusions (the Encoding Style Questionnaire). Following the reasoning presented above, we hypothesized that persons with the Internal (hasty) encoding style - who in the process of encoding are more likely to impose imperfect or even wrong encoding schemata - would be more likely to experience "split-second illusions" of identifying certain known objects or phenomena. Pilot studies revealed that people differ sharply in terms of how often they experience such "split-second" encoding errors.

For example, we asked college students in many classes, if, while driving down the highway, they have ever experienced "split-second" illusions of recognizing (erroneously) an animal moving on or off the road, only to find out a moment later that it was a piece of paper moved by the wind or a piece of an old tire.

We found marked differences in participants' responses: In a typical undergraduate class, approximately $30 \%$ of the students reported having this experience very often, while approximately $40 \%$ of the students reported having this experience "sometimes."

Interestingly, the remaining $30 \%$ of the students in a typical class deny ever having had this experience (some of them even expressed surprise that a "normal" person may have such a strange experiences and "see things that are not there...").

Based on the pilot studies and interviews with respondents, we identified nine types of real-life situations in which the phenomenon of the "split-second illusion" (or encoding error) occurs. These nine items asking about the relative frequency of having the respective experiences were mixed with 22 filler items (also asking about the relative frequency of specific experiences) and they formed the Encoding Style Questionnaire (labeled for participants "NISROE Questionnaire"). The crucial items are listed, below.

\begin{abstract}
2. When waiting on someone in the airport do you sometimes think that other people coming off the plane are the person you are waiting for? $1 \ldots 2 \ldots 3 \ldots 4 \ldots 5 \ldots 6$
\end{abstract}

5. Have you ever walked through the woods and thought for a split second that a piece of wood or a rock was an animal? $1 \ldots 2 \ldots 3 \ldots 4 \ldots 5.6$

13. For a split second from a distance do you sometimes mistake strangers for people you already know? $1 \ldots 2 \ldots 3 \ldots 4 \ldots 5 \ldots 6$

15. Sometimes when driving down the road do you see a piece of paper blowing in the wind, and for a split second think it might be an animal? $1 \ldots 2 \ldots 3 \ldots 4 \ldots 5 \ldots 6$

25. From a distance in a crowded parking lot, have you ever mistaken another car for your own? $1 \ldots 2 . .3 .4 .5 .6$

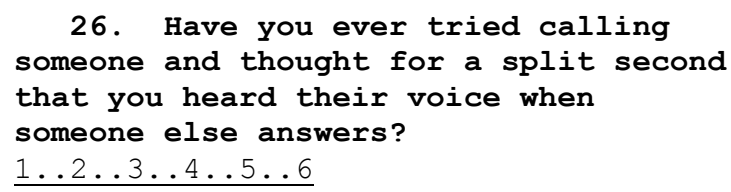

28. Have you ever seen a piece of rubber tire on the road while you were driving and for a split second thought it was an animal or something? $1 . .2 \ldots 3.4 .5 .66$

29. Have you ever seen a commercial or had a conversation about food and thought you could actually smell it? $1 \ldots 2 \ldots 3 \ldots 4 \ldots 5 \ldots 6$

31. Have you ever noticed something like a piece of paper just to the left or right of you and for a split second thought it was something else? $\quad 1 \ldots 2 \ldots 3 \ldots 4 \ldots 5 \ldots 6$

The analysis of data from over 200 participants who completed the questionnaire indicate that the nine items have relatively homogenous psychometric characteristics. 
The score for the "internal" (i.e., based more on "internal" categories as opposed to external data) encoding style was calculated as the average rating on the nine crucial items divided by the average rating on the remaining items (asking also about frequency of relatively "unusual" experiences); the higher the score, the higher the reported (relative) frequency of having experiences indicative of the 'hasty' application of encoding schemata.

An exploratory study with 73 participants did not reveal any reliable correlations between the measure of encoding style derived from the questionnaire, and the common personality dimensions measured by Myers-Briggs Type Indicator, Social Desirability scale

(Marlowe-Crowne); no correlations were found between the test score and a standard set of biographical data (including SES, family structure, etc.). Also no correlations were found with IQ or any of the scales of intelligence as measured by the Wechsler test (except for scales which involve visual information processing, where the scores of Internal encoders were higher).

The use of the terms "Internal" and "External" encoding style (in this chapter). In this chapter, we will use the terms "Internal" and "External encoding style." However, this does not mean that we assume any dichotomous typologies of encoding styles. At this point, it is safe to assume that the two "styles" represent ranges on a continuum (from "extremely internal" to "extremely external" encoding style). The distributions of both the scores from the Encoding Style Questionnaire and the related cognitive/experimental methods (that will be mentioned below) appear to be normal, or "flattened" normal.

The relation between the Encoding Style (as measured by the Encoding Style Questionnaire) and laboratory measures of self-perpetuation. Several experiments using cognitive research methods tested in the previous research on self-perpetuation have confirmed the expectation that those individuals who exhibit a relatively high level of selfperpetuation in the laboratory conditions are also those who experience split second illusions in everyday life more often (and they can be easily identified using the simple Encoding Style Questionnaire mentioned in the previous section).

(a) In one study, the Encoding Style Questionnaires (ESQ) were filled out by a group of 43 participants (college students) who then took part in a computer-controlled experiment ("Bamboo task"- see, Procedure number 1 in the Appendix: Example Procedures Used in Research on Self-perpetuation) designed to measure self-perpetuation of newly acquired encoding algorithms. Consistent with expectations, the self-perpetuating increase of the consistency of participants' responses with the newly acquired encoding algorithm was related to participants' scores in the $\operatorname{ESQ}(\underline{\mathrm{F}}(1,41)=4.54, \underline{\mathrm{p}}=.039)$. Specifically, participants who reported relatively higher frequencies of having the relevant experiences (hypothesized as indicative of the non-conservative, "hasty" (Internal) encoding style), also showed more self-perpetuation as measured in the computer-controlled cognitive task.

(b) This pattern was replicated in a follow up study with 29 different participants (this time, the $E S Q$ was filled out after instead of before the experimental task). When the 29 new participants were added to the initial sample, the reliability of the effect has increased further $(\underline{F}(1,70)=6.64, \underline{p}=.012)$.

(c) In a study with 62 participants (college students) using the "Brain scans procedure" (Lewicki, Hill, \& Sasaki, 1989; Lewicki, Hill, \& Czyzewska, 1994; Stamov-Rossnagel, 
2001, see also, Procedure number 2 in the Appendix: Example Procedures Used in Research on Self-perpetuation) to measure self-perpetuation, the rate of self-perpetuation again correlated reliably with the Internality index from the $E S Q(\underline{r}(60)=.32, \underline{p}=.005$, onetail).

(d) The same result was replicated in a study using the matrix scanning procedure (e.g., Hill, Lewicki, Czyzewska, \& Boss, 1989, Experiment 1; and Lewicki, Hill, \& Sasaki, 1989, Experiments 3a and 3b, see also, Procedure number 3 in the Appendix: Example Procedures Used in Research on Self-perpetuation) with 45 participants (college students), where the correlation between self-perpetuation and Internality was again in the expected direction and reliable $(\underline{\mathrm{r}}(43)=.36, \underline{\mathrm{p}}=.008$, one-tail $)$.

Tachistoscope and Partial Displays studies. In order to test the crucial - for the concept of Internal/External encoding styles - hypothesis that Internal encoders exhibit a lower (than External encoders) threshold of instantiation of interpretive schemata in the process of encoding, two studies were conducted. In the first one, 24 graduate students were exposed to tachistoscopic (and borderline subliminal) presentations of slides of everyday objects and asked to recognize them. As expected, Internal encoders (as measured by the $E S Q$ ) compared to External encoders were more accurate, $\underline{\mathrm{r}}(22)=.43, \underline{\mathrm{p}}=.018$ (Gill, 2000). In the second study (with 71 college students, conducted by Adrian and Gill, see Gill, 2000), the slides of objects were replaced with incomplete displays of letters made of fuzzy dots (and also displayed borderline subliminally). As expected, the accuracy of encoding (recognition of letters) was found to correlate with participants' encoding style $(\underline{\mathrm{r}}(69)=.37$, p. $=.0007)$, with the Internal encoders being more accurate. Also, this task allowed to register the response times and there was a trend found suggesting that the Internal encoders might have taken less time to identify the letters $(\underline{r}(69)=-.16, \underline{p} .=.08)$, however, the response time measure in this procedure was confounded by the speed of respondents' finding the proper key on the keyboard (e.g., typing skills or motivation).

Replication with seven-year old children. These studies (see the previous paragraph) were conceptually replicated with a sample of 52 seven-year old children (Brown, 1999). A version of the Encoding Style Questionnaire adjusted for this age group (e.g., "Have you ever thought that you saw an animal in the sky when it was really just a cloud?" "Have you ever been on the playground and thought that you saw one of your friends when it was really someone else?" "Have you ever been waiting for someone to pick you up from school (or a friend's house, etc.) and thought that you saw their car, but then it turned out to be someone else?"), was used and the index computed as before (i.e., the frequency of reporting split-second illusions relative to reporting other (unusual but irrelevant) experiences). The tachistoscopic images were changed to pictures from cartoons for children (e.g., Roadrunner, a rabbit, an owl). Again, those who were more Internal as measured by the modified $E S Q$ were also better at accurate encoding of the tachistoscopically presented images $(\underline{\mathrm{r}}(50)=.27, \underline{\mathrm{p}} .=.026$, Brown, 1999). Obviously, true longitudinal data would be necessary to support a claim about the stability of the cognitive style over the lifetime, but this preliminary result is at least consistent with that notion. (We are planning to collect follow-up data with the same participants next year (i.e., in 2004) when these participants - originally tested at the age of seven - will be 12-13 years old.) 
Cross-cultural replication (Lau, South Pacific). Another conceptual replication of this study was obtained with a sample of inhabitants of a small, isolated island in a remote part of the Lau archipelago in South Pacific, using a version of the Encoding Style Questionnaire adjusted for this group (e.g., "Have you ever thought for a moment that you saw a coconut crab when it turned out to be a piece of dark rock?" "Have you ever thought for a moment that you see a Nuqua [a black square-shape fish, commonly caught with a spear] only to realize it was just a weed or a shadow of something?"), and the tachistoscopic images changed to pictures of objects they were most familiar with (e.g., a bure, an octopus, a tabua, copra). Again, clear individual differences were found, and those who were more Internal as measured by the modified ESQ were also better at accurate encoding of the tachistoscopically presented images.

Specific skills as measured by real-life performance (sport studies). The hypothesized differences in the execution of encoding processes between Internal and External encoders (where the Internal encoders exhibit a lower threshold of instantiation of schemata that are imposed in the process of encoding) can produce testable hypotheses about the types of specific performance where the task requirements would benefit one encoding style over another.

Measures of specific aspects of performance in various disciplines of sport appear to provide good conditions to verify hypotheses about the theoretically expected advantages of Internal vs. External encoders across types of performance and we have collected supportive evidence in studies that focused on specific aspects of athletic skill acquisition and performance in tennis, soccer, and rowing. As expected, both External and Internal encoders were found to have specific advantages - different for each style - across different measured of their performance.

In one of these studies, which produced a particularly strong effect, Sandra Sailor (who was a psychology student and also a professional tennis instructor and player, Sailor \& Grubb, 1999) hypothesized that a particular skill that can be identified as a component of successful tennis playing can be facilitated by the Internal encoding style. Specifically, she noticed that one - not the decisive one but still important - component of being a good tennis player is related to the ability to anticipate the subsequent move of the opponent based on relatively ambiguous (and "probabilistic only") evidence such as the extremely subtle and usually impossible to articulate cues potentially generated by the opponent's body language. While this skill is not sufficient to be a winning player (which requires also the overall speed, stamina, tactics, etc.), this "anticipatory skill" is important and can be identified by experienced observers and especially well by those who play against the person to be appraised on this specific skill dimension.

Sailor had hypothesized that External encoders would be relatively less capable to quickly respond to such potentially useful cues - because their threshold of instantiation of schemata is higher - and thus this aspect of their game would be inferior to that of Internals. She recruited as participants for this study all nine members of the University of Tulsa, top women tennis team - a group of serious players, who treated tennis as an important part of their lives (most were supported by tennis scholarships), who trained together daily, played often against each other, and had reliable experience with each others' strengths and weaknesses as tennis players. Sailor had all of the them (a) fill out 


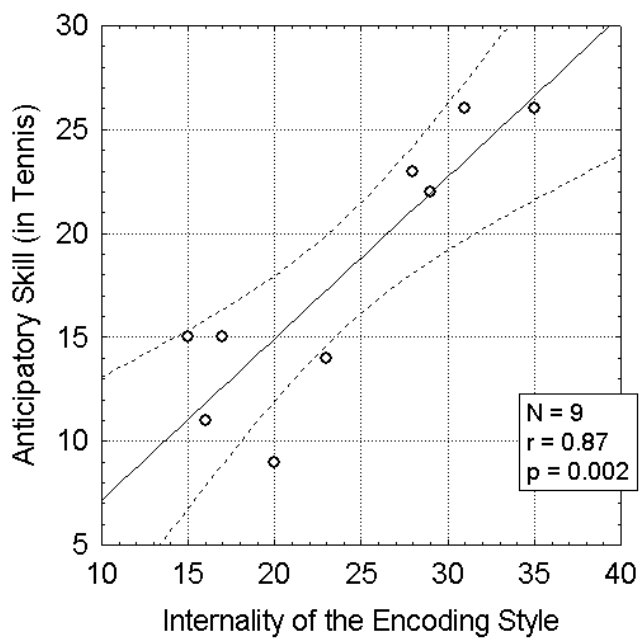

the Encoding Style Questionnaire and, at a different time (b) rate each other on the dimension of the anticipatory skill (as explained above), using a forced choice method where each player had to be qualified as belonging to the top (rating 3), middle (2), or bottom (1) group and the $\mathrm{N}$ in each of the three groups was supposed to be at least 2 . The sums of ratings obtained by each player were found to be highly correlated with the Internality of their encoding style $(\mathrm{r}(7)=.87, \mathrm{p}=.002)$. The anticipatory skill of virtually all four players who are Internal encoders was rated high and that of all five External encoders was rated low.

Evidence from the in-depth case studies. Gill and Phillips (Gill, 2000), conducted indepth cases studies of personalities of 16 persons selected (based on the results of the ESQ and other measures) as extremely Internal $(\mathrm{N}=8)$ and extremely External $(\mathrm{N}=8)$. The case study methodology involved three 1.5-hour, structured interview sessions (for a total of $4.5 \mathrm{~h}$, where participants' responses to 131 open-ended questions were recorded and later rated independently to produce quantitative scores), questionnaires, and experimental tasks. The extensive results of this study which cannot be reported here because of space considerations (they are described in Gill, 2000), can be fairly summarized as revealing significant, global (and also many specific) differences between personalities of these two extreme groups.

Among others, the results of the interviews provided further support for the notion that the Internal encoding style may un-specifically facilitate (through self-perpetuation) the development of biases contributing to (or associated with) symptoms of disorders (as confirmed before in the study by Hill, Lewicki, \& Neubauer, 1991), and this was further confirmed by the results of the NEO PI-R Inventory filled by the participants, where Internality of the encoding style correlated not only with Fantasy $(\mathrm{r}(14)=.75, \underline{\mathrm{p}}<.001)$, Feelings $(\underline{r}(14)=.67, \underline{p}<.004)$, and the Openness Domain $(\underline{r}(14)=.80, \underline{p}<.0002)$, but also with Anxiety $(\underline{\mathrm{r}}(14)=.52, \underline{\mathrm{p}}<.04)$, Depression $(\underline{\mathrm{r}}(14)=.57, \underline{\mathrm{p}}<.02)$, Impulsiveness $(\underline{\mathrm{r}}(14)=.71$, $\mathrm{p}<.002)$, and the Neuroticism Domain $(\mathrm{r}(14)=.58, \mathfrak{p}<.02)$. (Note that the values of these correlation coefficients may have been artificially inflated by the fact that the sample was not homogeneous but consisted of two extreme groups; however, they still indicate clearly different scores of the Internal and External encoders on the respective scales of the NEO Inventory.)

These case studies produced a large number of testable hypotheses about the personalities of Internal and External encoders, but among the most easy to test (and perhaps interpret) they produced convergent evidence (from both in-depth interviews and other measures) that across a spectrum of dimensions (personal life, work and vocational preferences, aesthetic preferences), External encoders prefer structured environments with clearly identifiable rules, while Internal encoders prefer less structured environments and do well in conditions of relative ambiguity that require reliance on intuition and making rapid 
decisions based on insufficient data.

This latter conclusion from the in-depth case studies which also appear to be consistent with the hypothesized core difference in the cognitive styles of Internal and External encoders, were tested in several studies where the actual preferences exhibited by participants in real-life conditions and their satisfaction with the relevant social arrangements were tested.

Preference for less constrained environments/activities. The predicted preference of Internal encoders for less constrained environments, was tested directly in a study conducted at the University of Tulsa MAC (Management Assessment Center), a unit that conducts research and provides services in the area of management training and assessment. Thirteen participants watched a training video tape of four persons ("assessees") taking part in so-called "leaderless group discussion" in an assessment center setting (created at the California State University, Fullerton, Student Assessment Center), and they were asked to rate the communication skill of the assessees.

Roughly a half of the participants first conducted the rating task by using the Coding Rules method, which was based on a set of detailed, and strictly defined rating rules, leaving very little freedom or interpretive ambiguity. The other half of the participants conducted the first rating task by using so-called Behaviorally-Anchored Rating Scales (BARS). The BARS method is much less algorithmic and restrictive and gave the participants more choice as to how to evaluate the performance of the assesses; in general, this method calls for a more global approach to the rating task. After a short break, the video was viewed again and the participants switched the rating methods. When the task was completed, the participants were asked to rate the ease of use of each tool on a scale of 1 - 10 (1 most difficult, 10 easiest).
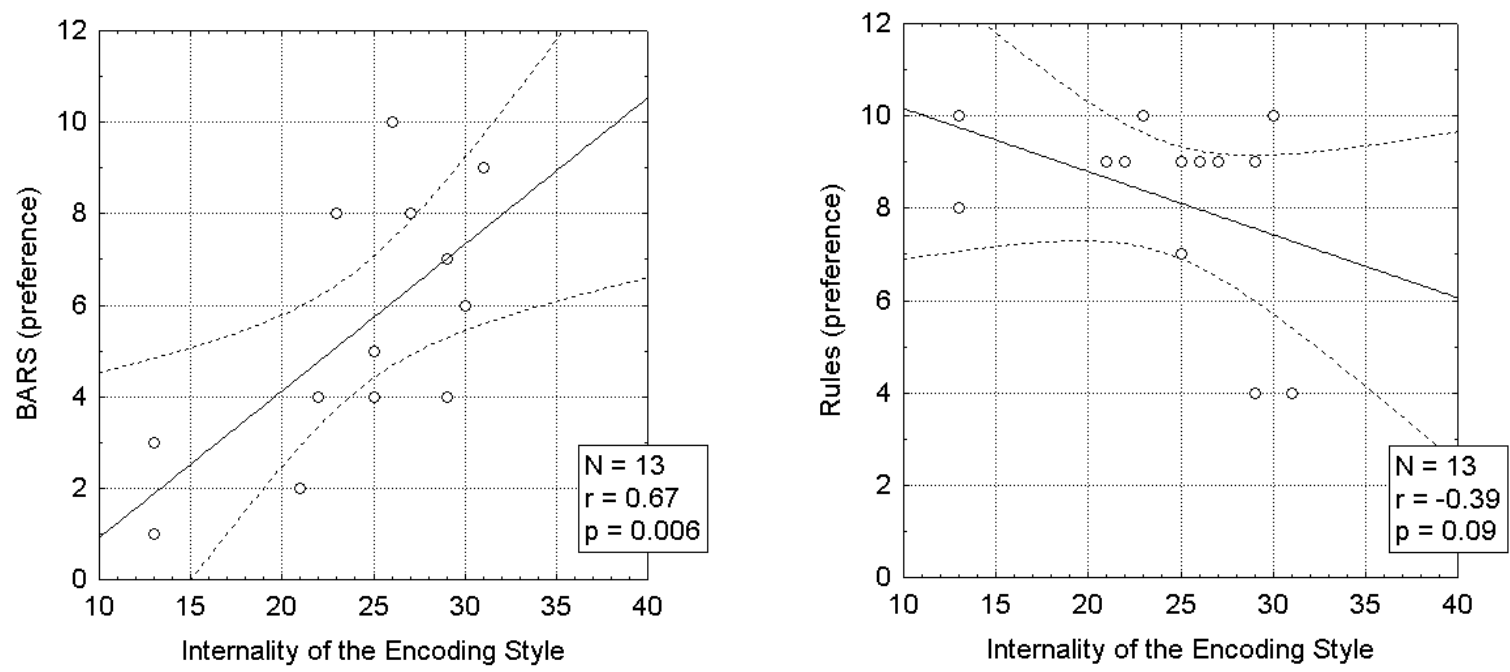

The results were consistent with the expectation that Internal encoders would prefer the less constrained (BARS) method over the rule bound Coding Rules method. The participants' scores of Internality correlated strongly with the preference for the BARS method $(\underline{r}(11)=.679, \underline{p}=.006)$; they also correlated negatively with the participants' preference for the Rules based method $(\underline{r}(11)=-39, \underline{p}=.09)$. 
Tolerance for new, unstructured environments. Following the reasoning presented above, concerning the relatively higher tolerance (if not a real preference) of Internal encoders for less structured environments - which involve ambiguity and where less conforming behavior is required or expected, we hypothesized that Internal encoders would relatively better adopt to new social environments (such as living in a foreign country).

We tested a large group of foreign students attending a school in the United states, thus who have experienced a transition to a new and more ambiguous (because less known) environment. Ascalon (2001) designed a comprehensive "International Students Survey" of the satisfaction (with different aspects of their lives) and invited to participate in this survey based study 250 foreign students who were attending University of Tulsa. The survey was relatively comprehensive and covered a wide variety of aspects of the university, social, cultural and personal life of the students. Most questions were based on 6-point rating scales and ranged from such topics as the quality of the curriculum to the respondents' perceived personal safety, attitudes towards America, and possible frustrations with any aspects of their lives. As many as 80\% of invited students (199 out of 250) participated in the survey (which was conducted over the campus Intranet) and as expected, Internal encoders turned out to be overwhelmingly better adjusted to life in the new - foreign for them - environment. While no similar patterns of correlations were found when the same questions were asked American students, foreign students who were Internal encoders turned out to be significantly more satisfied with virtually all aspects of their lives, perceived themselves as more successful in the new environment and more selfconfident. For example, foreign students who were Internal encoders felt more "comfortable about asking questions in class" $(\underline{\mathrm{r}}(197)=.55)$, "accepted by the American society" (.43), having friends (.42), being safe (.36), and better served by the school (.45). (All correlations significant at the $\mathrm{p}<.0001$ level.)

This study was also the first, other than the in-depth case studies summarize earlier, to provide support for the hypothesis that the style of encoding would determine vocational preferences, by facilitating both (a) different sets of specific cognitive skills, and (b) preferences for dealing with specific types of environments. Specifically, based on the theoretical reasoning discussed at the beginning of this chapter and also confirmed by the in-depth case study data (summarized earlier), it was hypothesized that external encoders would make real-life vocational choices in favor of jobs that involve more strictly rulebound activities (e.g., Programming, Engineering) while Internal encoders would chose jobs that are less rule-bound (e.g., Arts, Humanities).

Despite the very uneven distribution of majors chosen by members of this unusual sample (in which the majority of students who reported their majors, specialized in engineering or computer science - specifically, 149 out of 183), the hypothesis was fully supported. Specifically, the average Internality score of 35 participants who majored in nonengineering or computer related disciplines (such as art, humanities, or psychology) was 4.13 which was significantly higher than the mean of 3.23 achieved in the group of 149 engineering and computer science students $(\underline{\mathrm{t}}(181)=2.78, \underline{\mathrm{p}}=.003)$.

We found this result to be important because it demonstrated how the consequences of encoding style may translate into real choices and motivation (measured in an ecologically 
valid manner) to devote one's professional life to very different types of activities. Therefore, we decided to test this result in a series of two designated studies with more representative samples of participants (than the foreign, mostly engineering students tested before) and more even distributions of vocational choices made.

Vocational preferences of Internal and External encoders. The study was conducted by Osicki (2002) who tested the hypothesis that External encoders would make real-life vocational choices in favor of jobs that involve dealing with strictly rule-bound and conforming types of reality or environment (such as Computer Programming, Engineering (Electrical, Civil), or Accounting) while Internal encoders would chose jobs that are less rule-bound and conforming (Arts, Humanities, Business Management). These expectations received consistent and strong support in two studies with large, and highly representative samples of college graduates $(\mathrm{N}=146$ and $\mathrm{N}=345)$. Moreover, the data collected by Osicki (2002) demonstrate that even when the analysis was restricted from the broad spectrum of majors (e.g., from math to arts), to a much narrower range such as "Business School graduates only," the encoding style score (as measured by the ESQ) could still reliably predict the specialization area of the student - with External encoders choosing Accounting or MIS (Management Information Systems), and Internal encoders choosing more likely Business Administration or International Business.

\section{Summary and Conclusions}

The results of these studies demonstrate the role of cognitively interpretable (in terms of underlying mechanism), individual differences as the determinants of motivations that people demonstrate in their real lives.

The data provide consistent evidence for the existence of a dimension of encoding style that can be described in relatively specific terms of the underlying cognitive mechanisms confirmed by data collected using such indices as patterns of reaction times or rates of recognition of tachistoscopically presented stimuli - and which appear to determine a variety of interpretable behavioral consequences in important (also from the viewpoint of applied and not only basic research) areas ranging from social adjustment (e.g., perpetuation of symptoms of mental disorders) to specific cognitive skills and vocational preferences.

As suggested by the presented data, research on encoding styles can explain crucial, easily observable (and subjectively experienced) aspects of human behavior with low-level mechanisms identified using objective measures of cognitive performance in wellcontrolled laboratory conditions. The mere fact that something as concrete, observable, and "experiential" as the frequency of experiencing split-second illusions was found to be predictably correlated with objective measures (selected a priori) of low-level cognitive processes (such as patterns of RT or tachistoscopic indices) is encouraging and suggests that this phenomenon is worth further research attention.

In short, this phenomenon appears to be relatively unique in research on basic cognitive processes in that it not only deals with individual differences (an area of psychology which offers so far relatively few low-level, cognitive "explanations" or root causes of its subject matter, i.e., of the observable personality differences), but also, as suggested by the 
presented evidence, it may offer explanations of more than abstract personality traits, because it can help understand specific, observable behavioral choices made by people in their real lives (e.g., choices of careers).

\section{Appendix: Example Procedures Used in Research on Self-perpetuation}

General design of procedures to measure self-perpetuation of encoding dispositions. The experimental session (or sessions) of each procedure is normally divided into at least one learning phase and one testing phase. During the learning phase of each procedure, the participants are exposed to stimulus material containing a consistent but not salient (i.e., hidden) covariation between two features $\mathrm{x}$ and $\mathrm{y}$ (or more). In this phase, participants acquire procedural knowledge about the covariation, and this knowledge is expected to bias their encoding of new, relevant stimuli, as demonstrated in the earlier research (Lewicki, 1986a, 1986b, 1987; Lewicki, et al., 1992, 1994, 1997; Musen \& Squire, 1993; Seger, 1994; Stadler, 1989; Stamov-Rossnagel, 2001). For example, after participants learned about the relation between $\mathrm{x}$ and $\mathrm{y}$, a newly presented stimulus that is clearly $\mathrm{x}$ but ambiguous regarding $\mathrm{y}$, is likely to be encoded and perceived as being both $\mathrm{x}$ and $\mathrm{y}$. In the testing phase of each procedure, participants are exposed to a long series of stimuli that are relevant to the covariation between $\mathrm{x}$ and $\mathrm{y}$ learned in the first phase, in the sense that they clearly possess one of the features (i.e., they are unambiguous regarding feature $\mathrm{x}$ ).

However, they are neither consistent nor inconsistent with the covariation, because they are ambiguous (uninformative) regarding feature $y$. Thus, the testing phase stimulus material is open to the expected biased encoding (as consistent with the previously acquired $\mathrm{x}-\mathrm{y}$ encoding rule), although objectively, it is completely ambiguous regarding its factual consistency with the rule. Consistent with the self-perpetuation phenomenon, it is expected that the encoding bias acquired in the learning phase will gradually become stronger over the segments of the testing phase, that is, participants' encoding is predicted to become gradually more consistent with the covariation learned in the first phase. This general design was tested in numerous studies on self-perpetuation (e.g., Gill, 2000; Hill, Lewicki, Czyzewska, \& Boss, 1989; Hill, Lewicki, Czyzewska, \& Schuller, 1990; Hill, Lewicki, \& Neubauer, 1991; Lewicki, Hill, \& Sasaki, 1989, Lewicki, Hill, \& Czyzewska, 1994; Vequist, 2001).

Procedure 1: Evaluating the relative size of objects (the "Bamboo" task). In this procedure, the participant is presented on the computer screen with a colorful "bamboo stick" (a thick solid line made up of multiple colors); this object appears balanced on a pivot point that, during training, divides the bamboo stick into two uneven "halves." The participant' $\mathrm{s}$ task is to indicate as quickly as possible, to which side of the pivot the bamboo stick will tip, that is, which "half" of the bamboo stick is longer. The general procedure follows the standard design used in most of the previous experiments on selfperpetuation. In the training phase of the task, participants acquire an encoding algorithm (rule) relating the sizes of the bamboo-halves displayed on the computer screen with particular subtle aspects of the combinations of colors used in the color patterns of the 
lines. The self-perpetuation effect is measured by the increase in the consistency of participants' responses (with the learned rule) in the testing phase, where no supportive evidence is presented. In that testing phase, participants are exposed to a sequence of (ostensibly subliminal, but still visible) displays of lines (bamboo-sticks) balanced on the pivot point. Participants are asked to "guess" based on their "intuition" which one of the two parts of each line is longer (in fact, the lengths of the two halves are always identical). The guesses indicate whether participants follow the encoding rule acquired in the learning phase and whether the consistency of the responses with the rule gradually increases over time in the self-perpetuating manner (i.e., whether it increases despite the lack of any supportive evidence present in the testing phase material).

Procedure 2: Evaluating "brain scans." In the learning phase of this procedure (which was tested in previous studies, e.g., Lewicki, Hill, \& Sasaki, 1989; Lewicki, Hill, \& Czyzewska, 1994; Stamov-Rossnagel, 2001), participants are exposed to a series of complex graphics on a computer screen that are described as "digitized brain scans;" these brain scans are accompanied by personality descriptions of the scanned persons, ostensibly prepared by psychologists. The material contains nonsalient covariations between some hidden graphics features of the scans $(\underline{x})$ and personality descriptions $(\underline{y})$. In the testing phase, participants are exposed to a new series of scans (including the feature $\underline{x}$ ) and asked to make "intuitive judgments" of the manipulated personality characteristics (y). The "intuitive judgments" made by participants in the testing phase of the procedure follow the covariation between $\underline{x}$ and $\underline{y}$, and the consistency of the judgments with the rule gradually increases over time in the self-perpetuating manner (i.e., it increases despite the lack of any supportive evidence present in the testing phase material).

Procedure 3: Matrix-scanning paradigm. This procedure was used in numerous studies on acquisition of information about covariations (e.g., Lewicki, 1986a; Lewicki, Czyzewska, \& Hoffman, 1987; Stadler, 1989) and self-perpetuation (e.g., Hill, Lewicki, Czyzewska, \& Boss, 1989, Experiment 1; and Lewicki, Hill, \& Sasaki, 1989, Experiments $3 \mathrm{a}$ and $3 \mathrm{~b}$ ). In the training phase of the task, participants participate in a search task in which they speed-search a matrix of distractor characters (usually digits or letters) for a target character (usually digit 6 or letter $\mathrm{K}$ or $\mathrm{H}$ ). The manipulated covariations are built into the relations between target locations and features of the matrix of distractors. In the testing ("guessing") segment, the matrices are exposed for a very short time (ostensibly "subliminally"), and the participants are asked to "guess" the location of the target "following their intuition." Because in this phase, the matrices are hardly visible, participants cannot find out that these matrices in fact do not contain the target. Thus, if they have a "feeling of seeing" the target (and they see it in locations consistent with the pattern manipulated in the training phase), it can only be due to "inferences" they make in the process of encoding, by applying the encoding categories (covariations) learned in the training phase. The guesses of participants in the matrix scanning tasks indicate that the participants follow the encoding rules acquired in the training phase and that the consistency of the responses with the rule gradually increases over time in the selfperpetuating manner (i.e., it increases despite the lack of any supportive evidence present in the testing phase material). 


\section{Authors Notes}

Research reviewed in this chapter was partially supported by the National Institute of Mental Health Grant MH42715.

Address for correspondence: Pawel Lewicki, Department of Psychology, University of Tulsa, Tulsa, OK 74104; e-mail: pawel-lewicki@utulsa.edu.

\section{References}

Ascalon, E. (2001). Encoding Style and the Satisfaction of International Students (International Student Survey). Technical Report, University of Tulsa.

Brown, C. (1999). Internal and External Encoding Styles in Seven Years Old Children. Technical Report, University of Tulsa.

Cantor, N., \& Mischel, W. (1979). Prototypes in person perception. In L. Berkowitz (Ed.), Advances in experimental social psychology (Vol. 12, pp. 3-52). New York: Academic Press.

Combs, D. (in press). Implicit Learning and Non-clinical Paranoia: Does Content Matter? Personality and Individual Differences.

Gill, T (2000). Individual differences in schema activation, as measured by the Revised Nisroe (The Encoding Style Questionnaire). Unpublished doctoral dissertation, University of Tulsa, Tulsa.

Greenwald, A. G., Klinger, M. R., \& Liu, T. J. (1987). Unconscious processing of word meaning. Unpublished manuscript.

Greenwald, A.G. (1992). Unconscious cognition reclaimed. American Psychologist, $\underline{47}$, 766-779.

Greenwald, A. G. \& Banaji, M. R. (1995). Implicit social cognition: Attitudes, self-esteem, and stereotypes. Psychological Review, 102, 4-27.

Higgins, E. T., \& King, G. A. (1981). Accessibility of social constructs: Information processing consequences of individual and contextual variability. In N. Cantor \& J. Kihlstrom (Eds.). Personality, cognition and social interaction (pp. 69-121). Hillsdale, NJ: Erlbaum.

Higgins, E. T., King, G. A., \& Mavin, G. H. (1982). Individual construct accessibility and subjective impressions and recall. Journal of Personality and Social Psychology, $\underline{43}, 35-47$.

Hill, T., Smith, N., \& Lewicki, P. (1989). The development of self-image bias: A real world demonstration. Personality and Social Psychology Bulletin, 15, 205-211. 
Hill, T., Lewicki, P., Czyzewska, M., \& Boss, A. (1989). Self-perpetuating development of encoding biases in person perception. Journal of Personality and Social Psychology, 57, 373-387.

Hill, T., Lewicki, P., Czyzewska, \& Schuller, G. (1990). The role of learned inferential encoding rules in the perception of faces: Effects of nonconscious self-perpetuation of a bias. Journal of Experimental Social Psychology, 26, 350-371.

Hill, T., Lewicki, P., \& Neubauer, R. M. (1991). The development of depressive dispositions: A case of self-perpetuation of encoding biases. Journal of Experimental Social Psychology, 27, 392-409.

Hill, T., \& Lewicki, P. (1992). Personality and the unconscious. In V. J. Derlega, B. A. Winstead, \& W. H. Jones, (Eds.) Introduction to contemporary research in personality. Nelson Hall.

Kihlstrom, J. (1987). The cognitive unconscious. $\underline{\text { Science, } 237,1445-1452 .}$

Kunst-Wilson, W. R., \& Zajonc, R. B. (1980). Affective discrimination of stimuli that cannot be recognized. Science, 207, 557-558.

LaBerge, D. (1975). Acquisition of automatic processing in perceptual associative learning. In P. M. Rabbit and A. Dornic (Eds.), Attention and performance (Vol. V). London: Academic Press.

Lachman, R., Lachman, J. L., \& Butterfield, E. C. (1979). Cognitive psychology and information processing: An introduction. Hillsdale, NJ: Erlbaum.

Larkin, J. H. (1983). The problem of knowledge representation in physics. In D. Gentner \& A. L. Stevens (Eds.), Mental models (pp. 75-98). Hillsdale, NJ: Erlbaum.

Lewicki, P. (1983). Self-image bias in person perception. Journal of Personality and Social

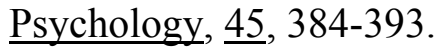

Lewicki, P. (1984). Self-schema and social information processing. Journal of Personality and Social Psychology, 47, 1177-1190.

Lewicki, P. (1985). Nonconscious biasing effects of single instances on subsequent judgments. Journal of Personality and Social Psychology, 48, 563-574.

Lewicki, P. (1986a). Nonconscious social information processing. New York: Academic Press.

Lewicki, P. (1986b). Processing information about covariations that cannot be articulated. Journal of Experimental Psychology: Learning, Memory, and Cognition, 12, $135-$ 146.

Lewicki, P., Czyzewska, M., \& Hoffman, H. (1987). Unconscious acquisition of complex procedural knowledge. Journal of Experimental Psychology: Learning, Memory, and Cognition, $\underline{13}, 523-530$.

Lewicki, P., Hill, T., \& Bizot, E. (1988). Acquisition of procedural knowledge about a pattern of stimuli that cannot be articulated. Cognitive Psychology, 20, 24-37. 
Lewicki, P., \& Hill, T. (1989). On the status of nonconscious processes in human cognition. Journal of Experimental Psychology: General, 118, 239-241

Lewicki, P., Hill, T., \& Sasaki, I. (1989). Self-perpetuating development of encoding biases. Journal of Experimental Psychology: General, 118, 323-337.

Lewicki, P., Hill, T., \& Czyzewska, M. (1992). Nonconscious acquisition of information. American Psychologist, 47, 796-801.

Lewicki, P., Hill, T., \& Czyzewska, M. (1994). Nonconscious indirect inference in encoding. Journal of Experimental Psychology: General, 123, 257-263.

Lewicki, P., Hill, T., \& Czyzewska, M. (1997). Hidden covariation detection: A robust and ubiquitous phenomenon. Journal of Experimental Psychology: Learning, Memory, and Cognition, 23 , 221-118.

McCord, M. (2002). Encoding Style and Perceived Difficulty of Rating Systems: "BARS" vs. "Coding Rules" for the Assessment Center. Technical Report, University of Tulsa.

Medin, D. L., \& Schaffer, M. M. (1978). A context theory of classification learning. Psychological Review, 85, 207-238.

Mischel, W. (1981). Personality and assessment. New York: Wiley.

Musen, G., \& Squire, L. R. (1993). Implicit learning of color-word associations using a Stroop paradigm. Journal of Experimental Psychology: Learning, Memory, and Cognition, $\underline{19}, 789-798$.

Osicki, M.A. (2002). Cognitive Determinants of Vocational Preference: The Internal/External Encoding Styles and Choice of Major. Unpublished doctoral dissertation, University of Tulsa, Tulsa.

Posner, M. I., Goldsmith, R., \& Welton, K. E., Jr. (1967). Perceived distance and the classification of distorted patterns. Journal of Experimental Psychology, $\underline{73}, 28-38$.

Reber, A. S. (1989). Implicit learning and tacit knowledge. Journal of Experimental Psychology: General, 118, 219-235.

Reber, A. S., \& Lewis, S. (1977). Implicit learning: An analysis of the form and structure of a body of tacit knowledge. Cognition, $\underline{5}, 333-361$.

Reed, S. K. (1972). Pattern recognition and categorization. Cognitive Psychology, $\underline{3}$, 382407.

Rumelhart, D. E. (1984). Schemata and the cognitive system. In R. S. Wyer, Jr. and T. K. Srull (Eds.), Handbook of social cognition (Vol. 1, pp. 161-188). Hillsdale, NJ: Erlbaum.

Rumelhart, D. E., \& Ortony, A. (1977). The representation of knowledge in memory. In R. C. Anderson, R. J. Spiro, and W. E. Montague (Eds.), Schooling and the acquisition of knowledge. Hillsdale, NJ: Erlbaum. 
Runeson, S., \& Frykholm, G. (1983). Kinematic specification of dynamics as an informational basis for person-and-action perception: Expectation, gender, recognition, and deceptive intention. Journal of Experimental Psychology: General, $\underline{112}, 585-615$.

Sailor, S. \& Grubb, M. (1999). The encoding style and the development of anticipatory skills in professional tennis players. Technical Report, University of Tulsa.

Schacter, D.L. (1996) Searching for Memory, New York: Basic Books.

Seger, C. A. (1994). Implicit Learning. Psychological Bulletin, 115, 163-196.

Smith, E. E., \& Medin, D. L. (1981). Categories and concepts. Cambridge, MA: Harvard University Press.

Stadler, M. (1989). On learning complex procedural knowledge. Journal of Experimental Psychology: Learning, Memory, and Cognition, 15, 1061-1069.

Stamov-Roßnagel, C. (2001). Revealing Hidden Covariation Detection: Evidence for Implicit Abstraction at Study. Journal of Experimental Psychology: Learning, Memory, and Cognition, 27, Number 4.

Sternberg, R. J. (1986). Intelligence applied. New York: Harcourt, Brace, Jovanowitch.

Sternberg, R. J. (1991). Metaphors of mind: Conceptions of the nature of intelligence. New York: Cambridge University Press.

Tellegen, A., Lykken, D. T., Bouchard, T. J., Wilcox, K. J., Segal, N. L., \& Rich, S. (1988). Personality similarity in twins reared apart and together. Journal of Personality and Social Psychology, 54, 1031-1039.

Tranel, D., \& Damasio, A. R. (1985). Knowledge without awareness: An index of facial recognition by propagnosics. Science, 228, 1453-1454.

Tulving, E., \& Schacter, D.L. (1990) Priming and human memory systems. Science, 247, 301-306.

Vequist, D. (2001). Acquisition and Self-perpetuating Development of Encoding Dispositions: A Flight Simulator Application. Unpublished doctoral dissertation, University of Tulsa, Tulsa.

von Hippel, W., Jonides, J., Hillon, J.L. \& Narayan, S. (1993) Inhibitory effect of schematic processing on perceptual encoding. Journal of Personality and Social Psychology, 64, 921-935.

Winograd, T. (1975). Computer memories: A metaphor for memory organization. In C. N. Cofer (Ed.), The structure of human memory. San Francisco: Freeman. 